\title{
Whose genomics?
}

A persistent Eurocentric bias in genomic studies means that advances in genomics research stand to benefit the few, not all. We need to change this.

W ith the completion of the Human Genome Project, this century has experienced a leap in understanding the genetics of human traits and diseases. However, the benefits of this research are not equal for everybody. Most of what we know about the human genome comes from and applies to a small proportion of the global population: individuals of European descent make up only $16 \%$ of the world's population, yet nearly $80 \%$ of participants in genomewide association studies (GWAS) are of European descent ${ }^{1}$.

An example of why it is important to look beyond European populations is published in this issue of the journal by Matoba and colleagues (https://www. nature.com/articles/s41562-019-0557-y). The authors investigate the genetic components of smoking behaviours in the Japanese population, comparing them to individuals of European descent. They find that the correlation of genome-wide genetic effects for smoking behaviours across their East Asian and European samples was significantly less than one $(\rho=0.72)$. Although most smoking-related lead single-nucleotide polymorphisms (SNPs) identified in the study were shared between the East Asian and the European samples, there were exceptions: two SNPs were found to have a high frequency in the Japanese population but a low frequency in the European population.

Tobacco use is the most important preventable lifestyle risk factor for many diseases, including respiratory diseases (such as chronic obstructive pulmonary disease), cancers (such as lung cancer) and heart disease. Currently responsible for 1 in 10 premature deaths worldwide ${ }^{2}$, smoking is a heritable but modifiable behaviour. Understanding the genetic aetiology of tobacco use can help identify and optimize strategies, interventions and drug targets for smoking prevention and cessation. Given the high mortality rates and the fact that more than a billion of the world's population smokes, this is clearly an area of significant public health concern. However, the vast majority of GWAS of smoking behaviours have been carried

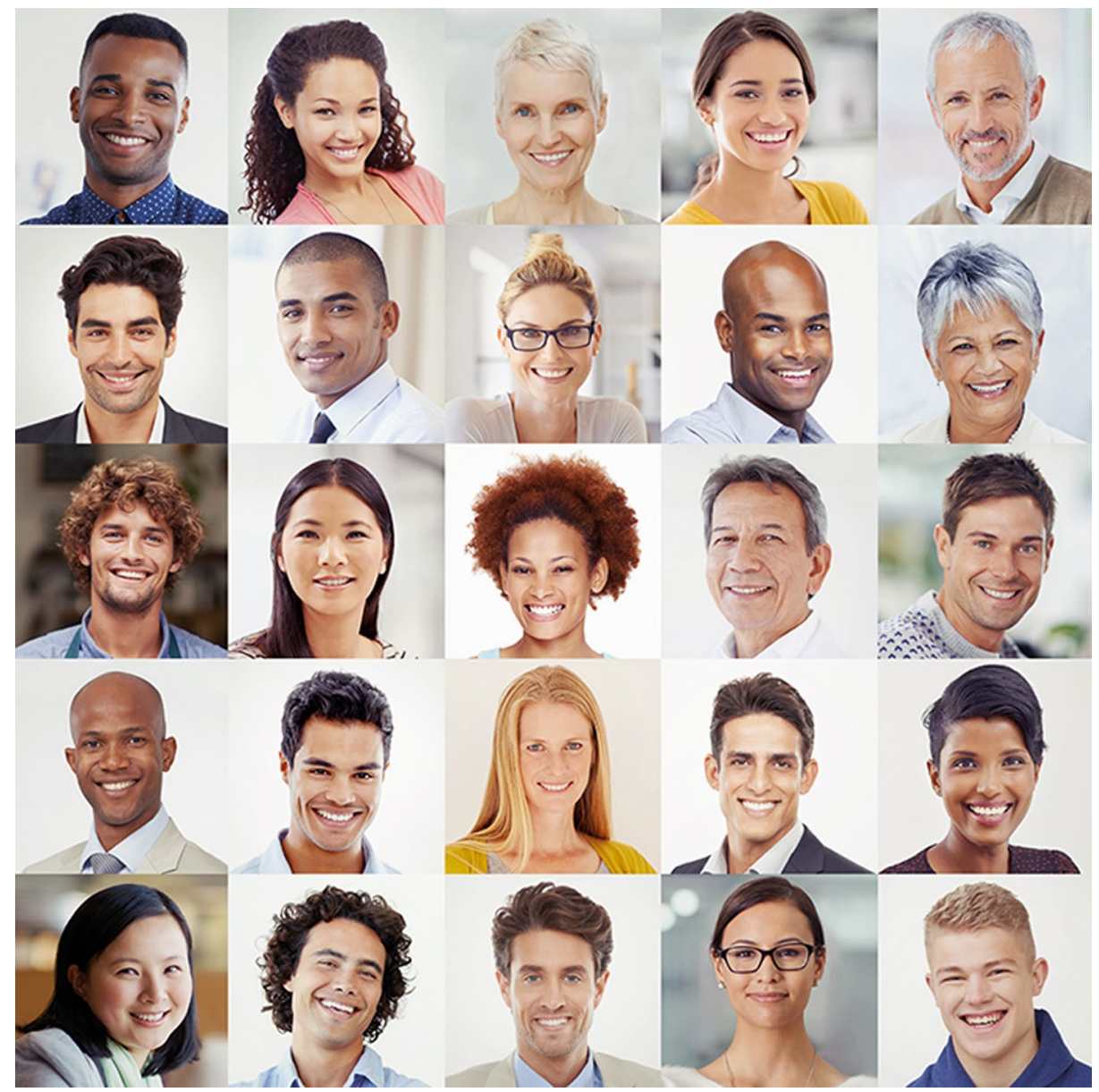

Credit: Yuri_Arcurs / E+ / Getty

out in participants of European descent. As a result, any genetically informed interventions or drug targets are likely only going to be optimal for people of European ancestry.

Genomic research has substantial potential to improve health outcomes. For instance, accumulating evidence suggests the potential clinical value of polygenic risk scores as biomarkers for disease prevention and better clinical care. However, it is people of European ancestry that can primarily or exclusively benefit from this research. Studies examining how well polygenic risk scores constructed out of GWAS data can be used to predict risk have invariably reached the same conclusion: polygenic risk scores predict individual risk far more accurately in people of European descent than in non-Europeans ${ }^{1}$. This is no surprise, as the data used to construct these scores come mainly from European participants.

Scientific research and precision medicine should not be for the benefit of a privileged minority of the world's population. Over the past decade, there have been repeated calls to address population imbalances in genomic research ${ }^{1,3,4}$. These calls have been accompanied by specific proposals on how to improve ethnic diversity in genomic research, both top-down and bottom-up. However, there has been very little progress in tipping the scales 
away from European samples ${ }^{1,4}$. We must recognize the ethical implications of this: genomic research, instead of

being a force for the good of all

humanity, may end up contributing to the maintenance and exacerbation of ethnic and socioeconomic disparities.

Published online: 14 May 2019

https://doi.org/10.1038/s41562-019-0619-1
References

$\square$ 1. Martin, A. R. et al. Nat. Genet. 51, 584-591 (2019).

2. Reitsma, M. B. et al. Lancet 389, 1885-1906 (2017).

3. Need, A. C. \& Goldstein, D. B. Trends Genet. 25, 489-494 (2009).

4. Popejoy, A. B. \& Fullerton, S. M. Nature 538, 161-164 (2016). 